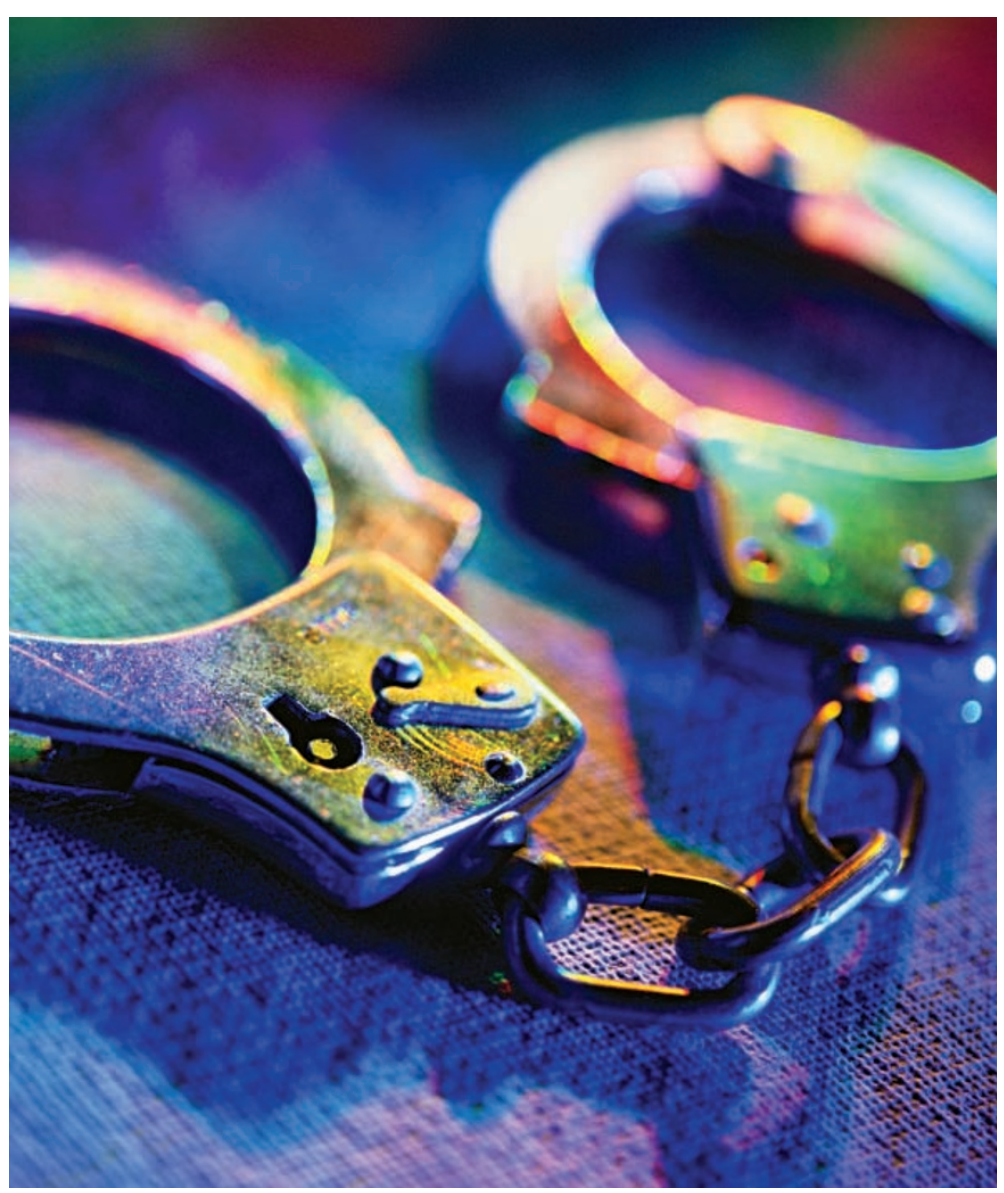

RNA-TARGETED DRUGS

\section{The ESSENCE of correction}

As many as half of the disease-associated single-nucleotide mutations in the coding regions of genes do not alter specific amino acids in the protein, but rather affect RNA splicing; for example, by inactivating or creating a splice site. And more often than not, these mutations result in the exclusion of exons from mRNA, a process known as exon skipping. So finding a way to correct these mutations and reinstate these exons into the transcript could be an effective route to treating the underlying cause of a wide range of diseases.

Now, in Nature Structural Biology, Adrian Krainer and Luca Cartegni describe the development of ESSENCE (exon-specific silencing enhancement by small chimeric effectors), which can correct these genetic typos by emulating the function of essential splicing factors called serine/arginine-rich (SR) proteins. SR proteins act at several steps during normal and alternative splicing they work by binding to sequences called exonic splicing enhancers (ESEs) through their RNA-binding domains and recruiting the cuttingand-pasting components of the splicing machinery through proteinprotein interactions mediated by an RS domain (a domain characterized by several Arg-Ser dipeptides).

To restore normal splicing in exonskipping models, Krainer and Cartegni fused a synthetic RS domain to an antisense fragment that binds to specific exons. They tested the ESSENCE concept first in the breast cancer 1 , early onset gene (BRCA1), in which a natural mutation in an ESE in exon 18 causes the exclusion of this exon from the mRNA. An ESSENCE compound containing the exon-18-specific antisense fragment restored accurate splicing in a dose-dependent manner in vitro, and required both the antisense-targeting fragment and the synthesized RS-protein-recruitment domain to carry out its function.

Krainer and Cartegni then looked at one of the best-characterized

\section{INFLAMMATION}

\section{Targeting two for the price of one}

Even after many years of research looking for new anti-inflammatory drugs, corticosteroids, such as dexamethasone, are still the main agents used to control inflammation. Although these drugs are very effective, their notable side effects keep the pressure high to develop safer antiinflammatories. Now it has been shown that inhibiting two enzymes in the inflammatory cascade with a single engineered peptide dramatically reduced inflammation in an animal model of inflammation, according to research in the January issue of The Journal of Clinical Investigation.

Most anti-inflammatory drugs inhibit the formation, or effects, of arachidonic acid metabolites, including prostaglandins, thromboxane and lipoxins, known collectively as eicosanoids. The phospholipase $\mathrm{A}_{2}\left(\mathrm{PLA}_{2}\right)$ family of enzymes catalyses the conversion of cell-membrane glycerophospholipids to arachidonic acid, and their inhibition could potentially block the synthesis of all eicosanoids. Corticosteroids induce many proteins, including the lipocortin and annexin families, whose anti-inflammatory activity can be explained, in part, by their ability to inhibit PLA P $_{2}$ Peptide sequences derived from these endogenous proteins are collectively referred to as antiflammins, and are recognized as one of the most potent classes of anti-inflammatory agents to date.

Some isotypes of PLA undergo posttranslational modifications by transglutaminases (TGases), which are multifunctional enzymes that catalyze intramolecular isopeptide bonds within $\mathrm{PLA}_{2}$ between specific lysine and glutamine residues or crosslink polyamines to glutamine residues. Sohn et al. began their studies by confirming that the activity of
TGases enhance the activity of PLA. Then, the authors designed a series of chimeric peptides that included a fragment of proelafin - a TGase substrate - together with the conserved core sequence of the antiflammin peptides. The new peptides inhibited both PLA ${ }_{2}$ and TGase activity. In a guinea-pig model of allergic conjunctivitis, one of these peptides was as potent as dexamethasone or antihistamine, on the basis of clinical inflammation scores, and was more potent in reducing eosinophil infiltration.

Although the mechanistic details of how these peptides work are not completely clear, these recombinant peptides are attractive candidates for clinical development as antiinflammatory agents.

Melanie Brazil

(2) References and links ORIGINAL RESEARCH PAPER Sohn, J. etal. Novel transgluatminase inhibitors reverse the inflammation of allergic conjunctivitis. J. Clin. Invest. 111, 121-128 (2003) FURTHER READING Miele, L. New weapons against inflammation: dual inhibitors of phospholipase A2 and transglutaminase. J. Clin. Invest. 111, 19-21 (2003) WEB SITE

Encyclopedia of Life Sciences: http://www.els.net Inflammation: acute | inflammation: chronic | phospholipases: degradation of membrane phospholipids 
examples of an ESE associated with disease: spinal muscular atrophy (SMA), a paediatric neurodegenerative disorder caused by the loss of both functional copies of the survival of motor neuron 1 (SMN1) gene. In this condition, the loss of SMN1 protein could be compensated by a related gene product called SMN2, but a single-nucleotide mutation in exon 7 of SMN2 produces an isoform that lacks this exon, resulting in a defective protein. The authors showed that creating an ESSENCE compound that targets this mutation restored the inclusion of exon 7 in the transcript in vitro. The technique is being developed to optimize in vivo delivery and activity, with the hope that the next generation of ESSENCE compounds will represent a viable approach for the treatment of SMA and many other genetic diseases.

Simon Frantz

(Q) References and links ORIGINAL RESEARCH PAPER Cartegni, L. \& Krainer, A. R. Correction of disease-associated exon skipping by synthetic exon-specific activators. Nature Struct. Biol. 10, 120-125 (2003) FURTHER READING Cartegni, L., Chew, S. L. \& Krainer, A. R. Listening to silence and understanding nonsense: exonic mutations that affect splicing. Nature Rev. Genet. 3, 285-298 (2002)

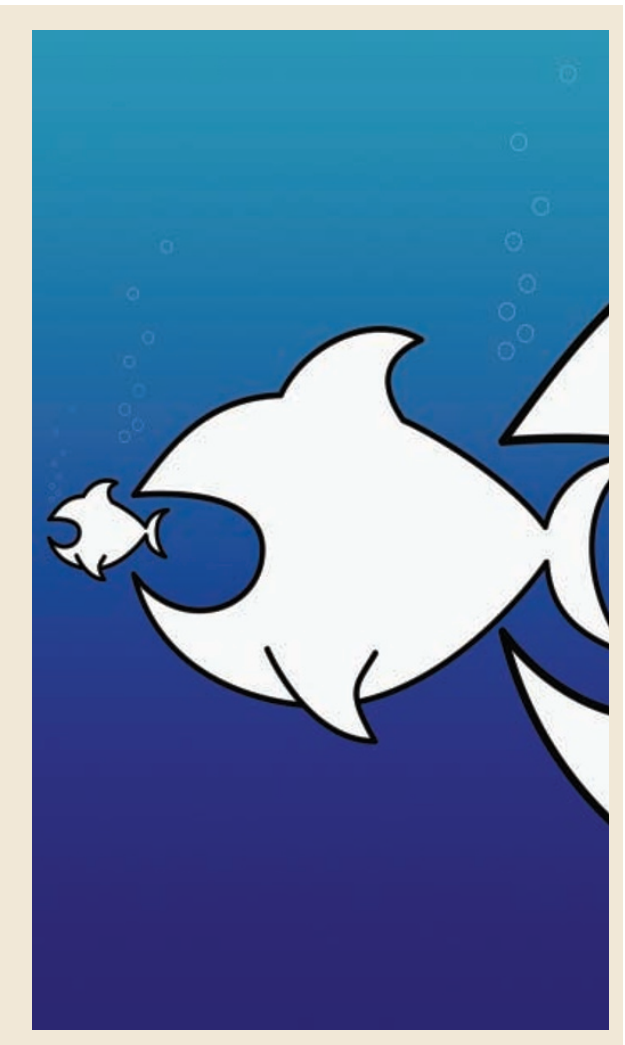

\section{STRUCTURE-BASED DRUG DESIGN}

\section{Can GPCR models work?}

Without doubt, the determination of the crystal structure of the G-protein-coupled receptor (GPCR) rhodopsin in 2000 was a landmark in the study of GPCRs. However, given the considerable challenges inherent in repeating this feat with other GPCRs, the key question for those involved in GPCR-based drug discovery is how well this structural information can be extrapolated to other GPCRs of therapeutic interest. As described in Proteins, Rognan and colleagues have tackled this issue, and show that models of a range of GPCRs based on the rhodopsin structure can be successfully used to identify both antagonists and agonists by virtual screening of compound libraries.

First, the authors constructed homology-based models of three well-studied GPCRs - the dopamine $\mathrm{D}_{3}$ receptor, the acetylcholine muscarinic receptor and the vasopressin $V_{1 a}$ receptor - by alignment with the rhodopsin sequence, mutation of the appropriate amino acids and then 'energy minimization' as a first step to optimize the protein conformation. To better model the potential 'antagonist-bound' state for each GPCR, a known antagonist was 'docked' into the active site using experimental binding data, and a further energy minimization performed.

Once the known antagonist was removed, the models were ready for testing. In each case, 10 known antagonists (but not those used in the previous energy minimization) were added to 990 drug-like compounds to give a virtual library of 1,000 compounds for each GPCR. These compounds were then docked into the appropriate GPCR model and their potential for binding assessed computationally. In all three cases, the procedure could successfully identify the known antagonists. For example, for the $\mathrm{D}_{3}$ receptor the optimum hit list of 37 potential binders contained seven of the known antagonists - a hit rate of $20 \%$, or 20 -fold higher than random screening of the full library would give ( 10 true hits out of 1,000 compounds). Importantly, an analysis of the structures of the ligands in the hit lists indicated that they were not biased by the antagonist used in the energy-minimization step, which means that it should be possible to retrieve new lead structures unrelated to any known GPCR ligand.

But would the procedure prove equally successful for modelling the 'agonist-bound' activated receptor state, which differs significantly from the 'antagonist-bound' receptor ground state, both in overall conformation and degree of

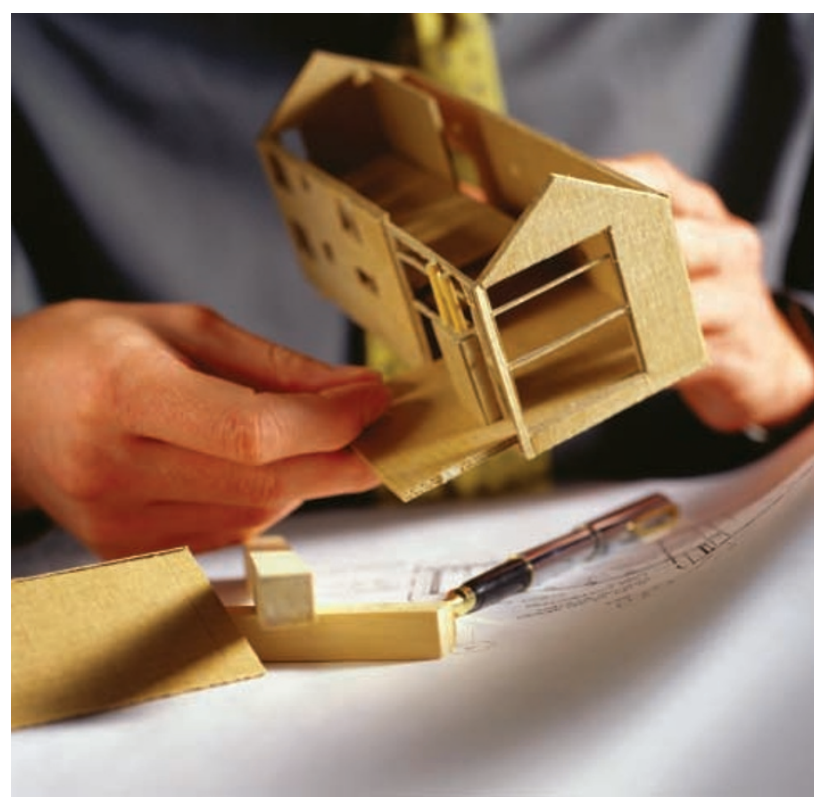

flexibility? After first making manual alterations to the GPCR structures assessed - the $\mathrm{D}_{3}$ receptor, the $\beta_{2}$ adrenoceptor and the $\delta$-opioid receptor to reflect the major conformational changes known to occur on receptor activation, the authors repeated the procedure used for antagonists, but also assessed a modified procedure to reflect the fact that there could be more than one 'activated' state for each receptor. Rather than just using one agonist to model the agonist-bound state, several different agonists were superimposed to generate a 'pharmacophore' model that should better represent the most important structural features of different agonist classes for the target receptors. Such pharmacophore models were then used in the energy minimization step, as before, to give a model of the agonist-bound state of each receptor.

An assessment of the hit lists after virtual screening of the 1,000-compound library for each receptor revealed that the pharmacophore-based models were significantly more successful than than those created by just single-agonist minimization, with hit rates comparable to those found with antagonists. For example, for the $\mathrm{D}_{3}$ receptor a hit rate of $30 \%$ was achieved, and again hits did not seem biased towards the ligand structures used in the generation of the receptor model. So, it seems that such approaches could be usefully exploited in searches for novel GPCR agonists and antagonists (once enough information is available on true ligands to allow model building), perhaps most profitably as an initial 'filtering' step to select the most promising compounds for experimental high-throughput screening.

Peter Kirkpatrick

(1) References and links

ORIGINAL RESEARCH PAPER Bissantz, C., Bernard, P., Hibert, M. \& Rognan, D. Protein-based virtual screening of chemical databases. II. Are homology models of G-protein coupled receptors suitable targets? Proteins 50, 5-25 (2003) 